\title{
Hidratación del silicato tricálcico en cementos mezclados elaborados con tobas cubanas
}

\section{Hidration of tricalcium silicate in blended Cements manufactured with cuban tuffs}

\author{
GABRIEL LUIS DUQUE FERNANDEZ, Laboratorio Central del Cemento \\ JULIO CESAR LLOPIZ YURELL, Universidad de La Habana \\ ESTER RUBIO FRIAS, CEADEN

\section{RESUMEN}

Se estudia la velocidad de hidratación del silicato tricálcico (alita) presente en el cemento con y sin adiciones, demostrándose que en presencia de tobas del yacimiento "Las Carolinas" en la provincia de Cienfuegos, Cuba, se acelera la velocidad de hidratación de este componente, obteniéndose constantes de velocidad de reacción aproximadamente 1,5 veces mayores que en el cemento patrón.

\section{SUMMARY}

The tricalcium silicate (alite) hydration velocity in cements with and without additives is studied in the presence of

"Las Carolinas" quarry tuffs from Cienfuegos province,

Cuba, the alite hydratrion velocity is enhanced reaching

velocity constants 1,5 higher than in the standard cement.

\section{INTRODUCCION}

La crisis energética renovó en muchos países el interés en la investigación y la producción de los cementos mezclados y como consecuencia de ello la necesidad de evaluar nuevos yacimientos de materiales puzolánicos (1).

En estudios previos de evaluación de la tobas del yacimiento "Las Carolinas" se encontraron relaciones estrechas entre la resistencia a compresión de los cementos mezclados y los valores de actividad obtenidos por el método de Fratini modificado, así como que estas tobas estaban compuestas parcialmente por ceolitas (Heulandita) más o menos contaminadas por calcita $(2,3)$.

Sersale R. et al $(4,5)$ detectó que los cementos mezclados elaborados con tobas ceolitizadas presentaban resistencias a compresión mayores a la edad de 28 días que los elaborados con puzolanas propiamente dichas. Atribuyendo este incremento de actividad a la existencia de canales que facilitaban la movilidad de los iones calcio hacia los sitios reactivos de la adición.

\section{INTRODUCTION}

The energy crisis has developed in multiple countries a renewed interest in blended cements research and production and as a consequence the need of developing new quarrys of pozzolanic materials (1).

In previous papers of "Las Carolinas" tuffs close relationships were found between the compresive strength of the blended cements and the values obtained from a modified Fratini method, and that this tuffs were partially zeolitized materials, being formed by heulandites contaminated in different extent with calcite $(2,3)$.

Sersale R. et al detected that tuffs gave higher compresive strength values at 28 days curing age than normal pozzolans $(4,5)$. Atributable to the zeolitic channels enabling enhanced movility to calcium ions helping in this way to increase reaction velocity. 
Con el fin de lograr una evaluación completa de la toba con su cemento se estudio la hidratación de la alita en pastas de cemento nezclado y patrón.

Yamaguchi G. y Tagaki S. (6) propusieron el máximo de difracción de la alita de distancia interplanar $1,76-1,78 \AA$ como el mejor en la investigación de la alita. Desde entonces en múltiples trabajos se ha demostrado que el mecanismo de hidratación de la alita es de difusión en una esfera que reduce su tamaño $(7,8,9)$. Otros trabajos muestran el efecto de la concentración de calcio en la hidratación de a alita $(10,11)$.

Como consecuencia de la reacción puzolánica hay una reducción del contenido de hidróxido de calcio en la pasta de cemento $(12,13)$ y uno de los objetivos del presente trabajo es estudiar si la presencia de las tobas afecta o no la velocidad de hidratación de la alita.

\section{MATERIALES Y METODOS}

Se tomaron dos muestras de tobas del yacimiento "Las Carolinas" de la provincia de Cientuegos, Cuba, que clasificaban como muy activas por el método de Fratini-modificado (2) $y$ un cemento de la Empresa cemento "Siguaney". La composición química de estas muestras aparece en la Tabla I.
In order to accomplish a complete evaluation of the tuff with its cement, the alite hydration was studied in blended and standard cement pastes.

Yamaguchi G. and Takagi S. (6) proposed the alite difraction maximum at 1,76-1,78 angstroms as the best for alite research. Since then numerous papers $(7,8.9)$ have shown that alite hydration is a shrinking core difusion controlled mechanism. Other papers show the effect of calcium concentration in alite hydration $(10,11)$.

As a consequence of pozzolanic reaction there is a reduction of calcium hydroxide contents $(12,13)$, and it is one of the objectives of this paper to show whether or not this affects alite hydration.

\section{MATERIALS AND METHODS}

Two tuffs samples of "Las Carolinas" quarry that, clasify as very active in the modified Fratini method /2/, and a cement of "Siguaney" cement plant were used. Their Chemical composition is show in table 1 .

\section{TABLA I (TABLE )}

Composición química de las tobas y del cemento utilizados (Chemical compositions of used tuffs and cement)

\begin{tabular}{|c|c|c|c|}
\hline Componente & $\begin{array}{l}\text { Cemento } \\
\% \text { en peso }\end{array}$ & $\begin{array}{c}\text { Toba } 1 \\
\% \text { en peso }\end{array}$ & $\begin{array}{c}\text { Toba } 2 \\
\% \text { en peso }\end{array}$ \\
\hline $\mathrm{SiO}_{2}{ }^{*}$ & 21,6 & 58,2 & 56,6 \\
\hline $\mathrm{Al}_{2} \mathrm{O}_{3}$ & 4,5 & 14,0 & 10.3 \\
\hline $\mathrm{Fe}_{2} \mathrm{O}_{3}$ & 4,3 & 5.9 & 5,0 \\
\hline $\mathrm{CaO}$ & 62,2 & 4,8 & 7.5 \\
\hline $\mathrm{MgO}$ & 1,7 & 2,3 & 1.9 \\
\hline P.F. & 1,7 & 11,5 & 14,7 \\
\hline $\mathrm{Na}_{2} \mathrm{O}$ & 0,27 & 1,55 & 0,90 \\
\hline $\mathrm{K}_{2} \mathrm{O}$ & 0,11 & 1,70 & 0,85 \\
\hline $\mathrm{SO}_{3}$ & 1,54 & - & - \\
\hline C.L. & 1,21 & - & - \\
\hline R.I. & 1.07 & - & - \\
\hline Total & 97,92 & 99,95 & 97,75 \\
\hline Frat-mod. & - & 13.1 & 10.0 \\
\hline \multicolumn{4}{|c|}{ Composición Mineralógica (FORMULAS DE BOGUE) } \\
\hline $\mathrm{C}_{3} \mathrm{~S}$ & $51,1 \%$ & & \\
\hline $\mathrm{c}_{2} \mathrm{~s}$ & $23,4 \%$ & & \\
\hline $\mathrm{C}_{3} \mathrm{~A}$ & $4,6 \%$ & & \\
\hline $\mathrm{C}_{4} \mathrm{AF}$ & $13.1 \%$ & & \\
\hline
\end{tabular}


Las tobas se molieron de forma que pasaran completamente por un tamiz de 150 micrómetros de luz; todas las mezclas se pesaron en balanza analítica y se mezclaron en un molino planetario de ágata por 10 minutos. El cemento patrón fue tratado de la misma forma, realizándosele a los cementos así obtenidos análisis granulométricos que muestran la no existencia de diferencias sustanciales en la granulometría de los cementos obtenidos, Tabla II.
The tuffs were milled as to pass completely through a 150 micrometers sieve. All mixes were weighed in analytical balance, mixing them in an agate planetary mill for 10 minutes. Any significant granulometric difference was detected as can be seen in table II.

TABLA II (TABLE II)

Granulometría de los cementos estudiados

(Studied cements granulometry)

\begin{tabular}{|c|c|c|c|}
\hline \multirow{2}{*}{$\begin{array}{l}\text { Abertura del tamiz } \\
\text { micrómetros } \\
\text { (Sieve opening } \\
\text { micrometers) }\end{array}$} & \multicolumn{3}{|c|}{ Retenidos en el tamiz en \% en peso (Residue in the sieve in \%) } \\
\hline & $\begin{array}{l}\text { Sin adición } \\
\text { (Without adition) }\end{array}$ & $10 \%$ Toba 1 & $10 \%$ Toba 2 \\
\hline 45 & 30 & 28 & 32 \\
\hline 30 & 40 & 39 & 41 \\
\hline 10 & 85 & 85 & 85 \\
\hline
\end{tabular}

Las pastas se elaboraron siguiendo el proceso descrito en (14) con una relación agua/cemento de 0,5, conservándolas las primeras 24 horas en cámara húmeda y el resto del tiempo de curado bajo agua en recipientes separados y sellados.

La hidratación se detuvo a la edad estudiada por el método propuesto en (14). Midiéndose el pico de la alita con distancia interplanar entre 1,76 y $1,78 \AA$ por difracción de rayos $\mathrm{X}$ en un equipo Dron III.

El agua fija y el contenido de Hidróxido de calcio se midieron termogravimétricamente.

\section{RESULTADOS Y DISCUSION}

Las áreas de los picos de alita se muestran en la Tabla III donde se puede ver un incremento en la hidratación de la alita desde los 21 días de curado. Los grados de reacción se muestran en la Tabla IV.
The pastes were made following the procedure described in /14/ with a water cement ratio of 0.5 , conserving them the first 24 hours in a moist cabinet and from that time on under water in separated sealed vessels.

Hydration was stopped at the ages studied by the procedure proposed in (14) and the alite peak between 1,76-1,78 angstroms of interplanar distance was measured by $X$ ray diffraction in a Dron III equipment.

The fixed water and calcium Hydroxide contents were measured thermogravimetrically.

\section{RESULTS AND DISCUSSION}

The alite peak areas are shown in table III where the alite enhanced hydration is observed in blended cements after 21 days curing time. The degrees of reaction are shown in table IV.

\section{TABLA III (TABLE III)}

Areas de los picos de alita a las distintas edades en $\mathrm{cm}^{2}$ (Alite areas at diferent ages in $\mathrm{cm}^{2}$ )

\begin{tabular}{|c|c|c|c|c|c|c|}
\hline \multirow{2}{*}{ Material } & \multicolumn{7}{|c|}{ Edad de curado en dias (Curing age in days) } \\
\cline { 2 - 7 } & $\mathbf{1}$ & $\mathbf{3}$ & $\mathbf{7}$ & 14 & $\mathbf{2 1}$ & $\mathbf{2 8}$ \\
\hline Cemento (Cement) & 40,95 & 31,13 & - & 28,33 & 23,67 & 18,93 \\
Cem.+10 \% Toba 1 (Cem.+10\% Tuff 1) & 34,55 & - & 24,32 & 23,24 & 18,88 & 13,61 \\
Cem.+10\% Toba 2 (Cem.+10\% Tuff 2) & 35,01 & - & 25,67 & 24,25 & 19,74 & 14,56 \\
\hline
\end{tabular}


TABLA IV (TABLE IV)

Grados de Reacción a las distintas edades

(Degrees of reaction at different ages)

\begin{tabular}{|l|c|c|c|c|c|}
\hline \multirow{2}{*}{ Material } & \multicolumn{5}{|c|}{ Edad de curado en dias (Curing age in days) } \\
\cline { 2 - 6 } & $\mathbf{3}$ & $\mathbf{7}$ & $\mathbf{1 4}$ & $\mathbf{2 1}$ & $\mathbf{2 8}$ \\
\hline Cemento (Cement) & 0,24 & - & 0,31 & 0,42 & 0,54 \\
Cem.+10\% Toba 1 (Cem.+10\% Tuff 1) & - & 0,30 & 0,33 & 0,45 & 0,61 \\
Cem.+10\% Toba 2 (Cem.+10\% Tuff 2) & - & 0,27 & 0,31 & 0,44 & 0,58 \\
\hline
\end{tabular}

El mejor ajuste entre todas las ecuaciones para esferas que se reducen controladas por la difusión para estos datos se obtiene por el modelo de Jander, en la cual la expresión $\left[1-(1-\alpha)^{1 / 3}\right]^{2}$ vs $t$ debe dar una línea recta.

Los resultados se muestran en la Tabla $V$ donde se puede observar que la constante de velocidad de reacción es aproximadamente 1,5 veces mayor que para el cemento patrón.
The best fit between all posible shrinking core difusion controlled mechanisms for this data is obtained by Jander modell in which the expression $\left[1-(1-\alpha)^{1 / 3}\right]^{2}$ vs $t$ should give a straight line.

The results are shown in table $V$ where it could be seen that the reaction velocity constant in the blended cements is nearly 1,5 times higher than for the standard cement.

TABLA V (TABLE V)

Resultados Cinéticos según Jander

[Kinetical results (Jander)]

\begin{tabular}{|l|c|c|c|}
\hline \multirow{2}{*}{$\begin{array}{c}\text { Parámetro estudiado } \\
\text { (Parameter studied) }\end{array}$} & $\begin{array}{c}\text { Sin adición } \\
\text { (none aditions) }\end{array}$ & $+10 \%$ Toba 1 & $+10 \%$ Toba 2 \\
\cline { 2 - 4 } & 0,00176 & 0,00272 & 0,00257 \\
\hline $\begin{array}{l}\text { Constante de velocidad de reacción } \\
\text { (Velocity constant) } \\
\begin{array}{l}\text { Coeficiente de correlacción } \\
\text { (Regres. coef.) }\end{array}\end{array}$ & 0,94 & 0,92 & 0,94 \\
\hline
\end{tabular}

Los contenidos de agua fija e hidróxido de calcio se muestran en las Tablas VI y VII respectivamente.

En la Tabla VI se puede observar que en todos los casos se incrementa el contenido de agua fija, pero desde los 14 días de curado es mayor el incremento en los cementos mezclados.
The fixed water and calcium hydroxide contents are shown in tables VI and VII respectivelly.

From table VI it could be noticed that in all cases the fixed water increase constantly, but after 14 days curing time there is a Higher increase in blended cements.

\section{TABLA VI (TABLE VI)}

Contenido de agua fija de las pastas de cemento en $\%$ (Fixed water contents of cement pastes in \%)

\begin{tabular}{|l|c|c|c|c|}
\hline \multirow{2}{*}{ Material } & \multicolumn{4}{|c|}{ Edad de curado en dias (Curirig age in days) } \\
\cline { 2 - 5 } & $\mathbf{7}$ & $\mathbf{1 4}$ & $\mathbf{2 1}$ & $\mathbf{2 8}$ \\
\hline Cemento (Cement) & 11,5 & 12,0 & 12,6 & 13,1 \\
Cem.+10\% Toba 1 (Cem.+10\% Tuff 1) & 12,6 & 13,4 & 14,0 & 14,6 \\
Cem.+10\% Toba 2 (Cem.+10\% Tuff 2) & 12,4 & 14,2 & 14,6 & 15,0 \\
\hline
\end{tabular}


Los contenidos de hidróxido de calcio muestran un incremento continuo en el cemento patrón, mientras que en los cementos mezclados disminuye a partir de los 14 días de curado, Tabla VII.
The calcium hydroxide contents show a continuos increment in the standard cement while in the blended cements it has a decremental concentration after 14 days curing age.

TABLA VII (TABLE VII)

Contenido de hidróxido de calcio en la pasta de cemento en $\%$ (Calcium hydroxide contents of cement pastes in \%)

\begin{tabular}{|l|c|c|c|c|}
\hline \multirow{2}{*}{ Material } & \multicolumn{4}{|c|}{ Edad de curado en dias (Curing age in days) } \\
\cline { 2 - 5 } & $\mathbf{7}$ & $\mathbf{1 4}$ & $\mathbf{2 1}$ & $\mathbf{2 8}$ \\
\hline Cemento (Cement) & 13,9 & 14,7 & 15,2 & 16,1 \\
Cem.+10 \% Toba 1 (Cem.+10\% Tuff 1) & 12,5 & 13,0 & 12,8 & 12,6 \\
Cem.+10\% Toba 2 (Cem.+10\% Tuff 2) & 11,3 & 12,1 & 11,8 & 11,7 \\
\hline
\end{tabular}

Estos dos resultados son un indicador cierto de que la reacción puzolánica comienza entre los 7 y los 14 días de curado.

Se puede observar una reducción mayor en el área del pico de la alita de los cementos mezclados por inspección simple de los datos a partir de los 21 días de curado, Tablas III y IV.

\section{CONCLUSIONES}

Los cementos mezclados con tobas de alta actividad muestran un incremento en la constante de velocidad de hidratación de la alita si se la compara con el cemento patrón. Se supone que la reducción en el contenido de hidróxido de calcio puede desplazar el equilibrio de la reacción.

La resistencia a compresión de los cementos mezclados se debe ver como una combinación de la reactividad de las tobas y la del cemento en su interacción mútua y no como la reactividad de unos y otros por separado.

El incremento en la velocidad de hidratación de la alita debida a la presencia de las tobas pudiera ser responsable, en parte, de la mayor resistencia que se observa en presencia de las mismas a la edad de 28 días.
These two results are a certain index of the beginning of the pozzolanic reaction between 7 and 14 days curing age.

A reduction in the alite peak area by $X$ ray diffraction can be seen by simple inspection after 21 days curing (tables III and IV).

\section{CONCLUSIONS}

Blended cements with high activity tuffs show an increase in reaction velocity constant for the alite hydration in comparison with a standard cement. It is supposed that the reduced calcium hydroxide contents displaces the reaction equilibrium (table $\mathrm{V}$ ).

The Compresive strength of the blended cements should be seen as a combination of the reactivity of the tuffs and that of cement and not only attributed to the higher activity of the former, taking into account that this enhanced activity has the complete responsibility of the enhanced alite hydration.

The enhanced alite Hydration could have a partial contribution in the higher compresive strength seen in tuff blended cements.

\section{BIBLIOGRAFIA}

(1) MASSAZZA F.: "Structure of Pozzolanas and Fly Ash and the Hydration of Pozzolanic and Fly Ash Cements". Tema IV, VII simposio de la química de los cementos, París 1980.

(2) DUQUE G. L., LLOPIZ J. C.: “Contribución a la normalización de una toba cubana". Ingenieria Estructural aceptado para publicación. 
(3) DUQUE G. L. LLPPIZ J. C., PUBIO E.: "Contribución a la normalizacion del uso de una toba cubana, parte Il". ingenieria estructural aceptado para publicación.

(-) SERSALE R.: "Structure and Characterization of Pozzolanas and of Fly Ashes" Subtema IV-1. VII Simposio de la nuimica de los cementos, Paris 1980.

(6) SERSALE R., FRIGIONE G.: "Fortland -Zeolite-- Cement for minimizing Alcail Agregate Expansion". Cenent ano Soncrete Research $17(3) 1987$ pp. 404-410.

(6) YAMAGUCHI G., TAKIG! S.: "Analysis of Portland Cement Clinker" $V$ Simposio de la quimica de los cementos Tokio 1968 Tomo I pp. $181+224$.

(7) KONDO R. YOSHIDA K.: "Miscibilities of Special Elements in Tricaicium Sllicate and Alite and the Hydration Poperties of $\mathrm{C}_{3} \mathrm{~S}$ Solid Solutions". $/$ Simposio de la quimica de los cemenios, Tokio 1968 , Tomolpp. $262-273$.

(B) DENT GLASSER L. S., LACHOWSKI E. E., MOHAN K., TAYLOR H. F. W: "A Multimethod Study of C 3 S Hydration". Cement and Concrete Research $8(6) 1978$ pp. $733-739$

(9) ODLER I, DORR H.: "Early Hydration of Tricaicium Silicate I. Kinetics of the Hydration Frocess and the Stoichiometry of the Hyaration Products" Cement and Concrete Researci $9(2) 1979$ po. $239-248$.

(1O) SKALNY J., JAWED I., TAYLOR H.F. W: "Studies on Hydration of Cement-Fiecent Developments". World Cement Technology $9(6)$ sep. 1978 pp. $183-193$.

(11) THOMAS N. L., DOUBLE D. D.: "The Hydration of Portiand Cement, $\mathrm{C}_{3} \mathrm{~S}$ and $\mathrm{C}_{2} \mathrm{~S}$ in the Presence of a Calcium Complexing Admixture (EDTA)". Cement and Concrete Research 13(3) 1983 pp. $391-400$

(12) MASSAZZA F.: "Chemistry of Pozzolanic Additions and Biended Cements". VI Simposio de la quimica de! cemento Moscú 1974 pp. 1-65.

(13) MUÑOZ CUSINE J. A., RABILERO BOUZA A.: "Cementos Puzoiánicos, su tabricación en Cuba". Construcción z(2) 983 pp. 7-34.

(14) DUQUE G. L., VELAZQUEZ J.: "Determinación del agua fija como método de control de resistencia a compresión en morteros de cemento sin adición". Ingenieria Estructural aceptado para puiblicación.

\title{
publicación del IETcc/CSIC
}

\author{
ACUEDUCTOS ROMANOS EN ESPAÑA \\ Carlos Fernández Casado \\ Prof. Dr. Ing. de Caminos, Canales y Puertos
}

Esta publicación se compone de una serie de articulos, publicados en la Revista "Informes de la Construcción", en los cuales se hace un análisis de ios acueductos romanos que existen en España y el balance de las condiciones de conservación en que se encuentra cada uno de ellos, incluyendo referencias históricas y literarias.

Se ha ilustrado con la reproducción de la valiosa documentación gráfica que posee el prestigioso autor.

Un volumen encuadernado en couché, a dos colores, de $21 \times 27$ centímetros, compuesto de 238 páginas, numerosos grabados, dibujos, fotos en blanco y negro y figuras de línea.

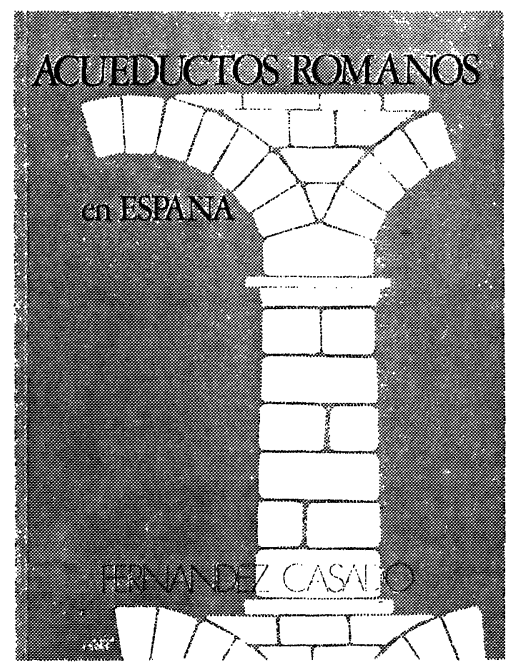

Precio: España, 900 ptas., $13 \$$ USA. 